\title{
Deutsch als Fremdsprache - Definition und Abgrenzung eines wissenschaftlichen Faches
}

\author{
Brigitte Sorger
}

Political and social changes affect the motives for learning German and have a lasting impact on the professional and educational profile. The article outlines the new challenges, the need to extend the related sciences and the new focus in curricula, at conferences and in professional literature. Thus could make German as a foreign language a practice-relevant study and interdisciplinary research subject.

German worldwide - language policy - lines of development - related sciences - curricula of education

Politische und gesellschaftliche Veränderungen beeinflussen die Motive Deutsch zu lernen nachhaltig und wirken sich dadurch auf das Fach- und Ausbildungsprofil aus. Der Beitrag skizziert die neuen Herausforderungen, die erforderliche Ausweitung der Bezugswissenschaften und die neue Schwerpunktsetzung in Curricula, bei Konferenzen und in der Fachliteratur, die Deutsch als Fremdsprache heute zu einem praxisrelevanten Studium und interdisziplinären Forschungsfach machen können.

Deutsch weltweit - Sprachenpolitik - Entwicklungslinien - Bezugswissenschaften Ausbildungscurricula

\section{Einführung}

Die Lehr- und Lernsituation für die deutsche Sprache hat sich in den letzten zwanzig Jahren in der Tschechischen Republik grundlegend geändert. Während nach der politischen Wende Deutsch in manchen Grenzregionen noch die wichtigste und meistgelernte Fremdsprache war, setzt sich trotz Mehrsprachigkeitskonzepten der Europäischen Union, die etwa als erste Fremdsprache lieber die Nachbarsprache vermittelt sehen wollen, aktuell das Englische auf allen schulischen Ebenen durch. Dies hat natürlich auch Konsequenzen für die Ausbildung der Lehrkräfte, da diese sich nun stärker mit Tertiärsprachenkonzepten befassen und die Ziele des Fremdsprachenunterrichts neu definieren müssen. Neue Ziele und Konzepte bedürfen aber auch eines kritischen Blicks auf die Bezugswissenschaften eines Faches ebenso wie auf das Selbstverständnis, welche Schwerpunktbereiche sich das wissenschaftliche und das Lehrfach geben wollen.

\section{Deutsch weltweit}

Obwohl die deutsche Sprache vorrangig in Europa gesprochen wird, ist sie doch eine weltweit verbreitete Fremdsprache. Etwa 100 Millionen MuttersprachlerInnen 
(DaM) in den DACHL-Ländern, in bilingualen Regionen (etwa in Südtirol, Rumänien, Brasilien u. a.) und in Regionen, wo Deutsch als Minderheitensprache nach wie vor gesprochen wird (z. B. in Kasachstan, Namibia, Tschechien etc.), stehen nach Schätzungen allein in der Europäischen Union 55 Millionen Menschen gegenüber, die Deutsch als Fremdsprache sprechen (EUROPÄISCHE KOMMISSION: 2006, 8). Zu den Ländern mit dem höchsten Anteil gehören dabei Ungarn, wo etwa ein Viertel der Bevölkerung Deutsch kann, und Tschechien mit beinahe einem Drittel (EUROPÄISCHE KOMMISSION: 2006, 4). Anderen Erhebungen zufolge sind es etwa $27 \%$ aller Bewohner in den Mittel-Osteuropäischen Ländern (HERNIG: 2005, 30), aber auch in Ländern wie den USA gibt es etwa 7,5 Millionen Deutschsprecher (McCOMB), in Russland vermutlich um die 10-20 Millionen und selbst in Japan hat Deutsch einen hohen Stellenwert. Zählt man zu den Deutsch als Fremdsprache-Sprechern (DaF) noch die MigrantInnen, die ja Deutsch als Zweitsprache (DaZ) nutzen, hinzu, so übertrifft die Sprecherzahl weltweit sicherlich die der Muttersprachler. Wirklich genaue Angaben hierzu existieren aber nicht, denn im Normalfall erheben die Befragungen die Lernerzahlen. Hier wiesen bereits die ersten weltweiten Erhebungen, die die BRD in den sechzigerund siebziger Jahren des 20. Jahrhunderts vornahm, Zahlen um die 22 Millionen LernerInnen in 61 Ländern aus (AMMON: 1989, 256). ${ }^{1}$ Dem standen andere Schätzungen gegenüber, nach denen alleine in der UdSSR in den siebziger Jahren rund 20 Millionen Deutschlerner zu verzeichnen waren (HEXELSCHNEIDER: 1980, 136). 1985 wies die BRD in einer Studie schulischen Deutschunterricht in 88 Ländern und an Hochschulen in 91 Ländern nach (BUNDESREGIERUNG: 1985). Wirklich seriöse Daten kann man allerdings erst seit den STADaFErhebungen annehmen, nach denen im Jahr 2000 weltweit etwa 18 Millionen Menschen Deutsch lernten (SATORIUS/RUCKTESCHELL: 2000, 75) und 2005 von 160 Staaten weltweit in 135 Deutsch als Fremdsprache gelehrt wurde (StADaF: 2005).

Die aktuellsten Daten aus dem Jahr 2010 weisen 141 Staaten aus, in denen Deutsch gelernt wird, wobei die Lernerzahl allerdings seit 2005 rückläufig ist, wie das Goethe-Institut in seinem Kommentar zu den Erhebungen auch explizit ausführt:

2010 verzeichnete diese Statistik insgesamt 14.042.789 Deutschlerner. Das sind 2.675.912 weniger als 2005. Einen großen Rückgang gab es vor allem in den Ländern der ehemaligen Ostblockstaaten, wo noch bis Anfang des Jahres 2000 die Nachfrage nach Deutsch ungewöhnlich hoch war. (FIEBACH: 2010)

1 Ammon zitiert hier die Stellungnahme der Bundesregierung zum Bericht der Enquet-Komission, 1977. 
Zu den Ländern mit den stärksten Rückgängen zählte auch 2010 Tschechien, nachdem es im STADaF-Bericht ${ }^{1}$ von 2005 bereits explizit als Beispiel für stark fallende Lernerzahlen hervorgehoben worden war (StADaF: 2005, 14). Von 2000 bis 2005 sanken die schulischen DaF-Lernerzahlen um 127.000 (auf 496.000) und die an Hochschulen um 89.000 (auf 35.000) (StADaF: 2005, 14). Bis 2010 sank die Lernerzahl an den Schulen nochmals um 132.000 auf 364.000, im Hochschulbereich aber war wieder ein Anstieg um 8.000 Deutsch-Studierende zu verzeichnen, sodass aktuell 43.000 Deutsch-Studierende in Tschechien ausgewiesen sind (NETZWERK DEUTSCH: 2010). Diese unterschiedliche Entwicklung an Schulen und Hochschulen lässt sich zum Teil mit der tschechischen Sprachenpolitik erklären, die ja bekanntlich unter Mehrsprachigkeit eine starke Forcierung des Englischen versteht und deshalb an den Schulen vorrangig entsprechende Fördermaßnahmen setzt. Die wieder steigenden Studierendenzahlen aber belegen, dass die Bedürfnisse der Gesellschaft, der Wirtschaft und des Individuums ganz andere sind.

\section{Gründe für das Erlernen der Fremdsprache Deutsch}

Helmut Glück meint, „das Bedürfnis, das Deutsche als Fremdsprache zu lernen, gibt es genau so lange, wie es das Deutsche als Sprache gibt" (GLÜCK: 2002, 1) und tatsächlich kann man seit dem Mittelalter speziell in Nordeuropa, Ungarn und im west- und südslawischen Raum die deutsche Sprache als die wichtigste (moderne) Fremdsprache bezeichnen. Bereits im 15. Jahrhundert lassen sich erste Lehrmaterialien in Italien nachweisen und die ältesten bekannten gedruckten Materialien gab es im 16. Jahrhundert in Böhmen, Ungarn, Frankreich, Katalonien und Italien (GLÜCK: 2002a).

In früherer Zeit waren die Motive eine fremde Sprache zu erlernen durchaus pragmatische, man benötigte Sprachen für den Fernhandel, „Fernheiraten“ und Kavaliersreisen (LEHBERGER: 2003, 609 f.), aber auch Flucht und Migration zwangen zum Erwerb einer neuen Sprache. Lernte der Adel eher mit Unterstützung eines „Sprachmeisters“, so wurde der „Kinderwechsel“ in bürgerlichen und sogar bäuerlichen Bevölkerungsgruppen gezielt als Möglichkeit zum Erwerb einer anderen (Landes-)sprache eingesetzt (EDER: 2006, 76). Gesetzliche Sprachregelungen unter Maria Theresia und Joseph II. machten schließlich Deutsch nicht nur zur Beamtensprache und Kommandosprache beim Militär, sondern initiierten auch ein gezieltes schulisches System zur Förderung der deutschen Sprache (vgl. EDER: 2006). Aber auch im Deutschen Reich wurde die bewusste Verbreitung der deutschen Sprache nicht nur in den Kolonien durch den 1878 eingerichteten „Reichsschulfond“ gefördert. Im Jahr 1914 konnten Kinder bereits an 900 deutschen Auslandsschulen

12005 nannte sich die erhebende Gruppe noch STADaF (Ständige Arbeitsgruppe Deutsch als Fremdsprache), 2010 war sie in Netzwerk Deutsch umbenannt worden. 
die deutsche Sprache erlernen, auch wenn diese Schulen noch stark den deutschen Minderheiten vorbehalten waren (KRATH: 2008, 20).

Seit dem Zweiten Weltkrieg hat sich bekanntlich generell das Lernen lebender Fremdsprachen nicht nur methodisch, sondern auch zahlenmäßig stark verändert. Die moderne Gesellschaft und die Globalisierung haben den Trend verstärkt, sodass Fremdsprachenlernen auch zum Politikum geworden ist und sich in zahlreichen sprachenpolitischen Dokumenten widerspiegelt. Während noch von den fünfziger bis in die achtziger Jahre des Zwanzigsten Jahrhunderts die Beherrschung einer Fremdsprache eine Frage der höheren Bildung war, fordert die Europäische Union heute für alle BürgerInnen die Kenntnis von zwei Fremdsprachen. Entsprechend haben sich auch die Lernmotive verändert, wie die letzte Studie der Europäischen Kommission belegt, denn generell werden „die Gründe für das Erlernen von Fremdsprachen im Vergleich zu den Ergebnissen vor vier Jahren immer stärker mit praktischem Nutzen verknüpft, etwa um die Sprachkenntnisse für die Arbeit zu nutzen (32\%) oder um im Ausland zu arbeiten $(27 \%)$ " (EUROPÄISCHE KOMMISSION: 2006, 5).

Die Intensivierung des Sprachlernens und die Neudefinition der Motive aber haben auch zu einer Veränderung der Vermittlungsmethode geführt. Während sich die Grammatik-Übersetzungmethode noch am Lerner der klassischen Sprachen orientierte, passten sich die weiteren Methoden nicht nur den technischen Neuerungen, sondern auch den pragmatischen Sprachlernmotiven an. Die heutige Methodik berücksichtigt, dass LernerInnen mehrere Sprachen parallel erlernen, diese allerdings auf unterschiedlichem Niveau, da für unterschiedliche Zwecke. Im Mittelpunkt steht die Sprachanwendung, nicht mehr die Sprachbeherrschung und das metasprachliche Wissen. Dies wiederum hat elementare Auswirkungen auf die Ziele mit denen an Schulen Sprachen erlernt werden und nicht zuletzt auf die LehrerInnen und deren Ausbildung. Daraus folgt der Bedarf einer Neuorientierung für das Studienfach Deutsch, das sich stärker aus seiner lingustischliteraturwissenschaftlichen Fixierung lösen sollte und müsste, um dem neuen Berufsbild eines „Deutsch-Absolventen“ gerecht werden zu können.

Auch Werner Roggausch, der ehemalige Leiter der Gruppe „Germanistik und Deutsche Sprache im Ausland" beim Deutschen Akademischen Austauschdienst (DAAD), stellt fest: „In fast allen Ländern geht die Nachfrage nach einer solchen germanistischen Philologie zurück“ (ROGGAUSCH: 2008, 6). Bedingt durch weltweite Veränderungen und verstärkt durch die Bologna-Reformprozesse erwarten die Studierenden heute eine Qualifikation für eine berufliche Perspektive. Roggausch unterlegt den Trend auch mit Beispielen: „In Schweden etwa wurden germanistische Studiengänge wegen Mangel an Studenten geschlossen, obwohl dort die Nachfrage nach Deutsch generell erkennbar zunimmt" (ROGGAUSCH: 2008, 7). Ähnliches verzeichnetauch das Goethe-Institut: „In den Feldern Lehrerausbildung, Dolmetscherund Übersetzerausbildung, Medien, Sprachausbildung für Wirtschaft, Jura oder 
Tourismus nimmt die Nachfrage eher zu“ (GIERSBERG: 2009). Im Gegensatz zu diesen konkreten berufsorientierten Studienzielen aber sei die Auslandsgermanistik „jedoch vielfach noch deutlich von einer klassisch philologischen Ausrichtung geprägt. Im Mittelpunkt dieser traditionell bildungsorientierten Studiengänge stehen Deutsche Literatur und Sprache oder Sprachgeschichte" (GIERSBERG: 2009).

\section{Neue Bezugswissenschaften des Deutschen als Fremdsprache}

Ein verändertes Ausbildungs- und Berufsziel erfordert natürlich ein ebenfalls verändertes Ausbildungscurriculum. Dieses aber gestaltet sich ausgehend von den ebenfalls neu einzubeziehenden Bezugswissenschaften. Da im Zentrum des Interesses nach wie vor die deutsche Sprache steht, bleiben die germanistische Linguistik und die Literaturwissenschaft grundlegende Bezugswissenschaften. Sie sind aber mit einer Neudefinition des Faches dringend zu erweitern. Das Fachlexikon Deutsch als Fremdsprache definiert die Disziplin wie folgt: „DaF bezeichnet alle unterrichtspraktischen und wissenschaftlichen Aktivitäten, die sich mit der deutschen Sprache und Kultur der deutschsprachigen Länder unter dem Aspekt des Lernens von Nichtdeutschsprachigen beschäftigt" (FACHLEXIKON: 2010). Logischer Weise sind damit einzubeziehen: die Geschichte, Politologie, Kulturgeographie und Ethnologie als Bezugswissenschaften des landeskundlichen Lernens, aber auch die Sprachlehrforschung, die Fremdsprachendidaktik, die allgemeine Pädagogik bzw. die Bildungsforschung und schließlich die Spracherwerbsund Gehirnforschung. Entsprechende Forderungen werden seit einigen Jahren nicht nur für das Hochschulfach (vgl. z. B. HERNIG: 2005, 65) oder von Fachverbänden $(\mathrm{FaDaF})$ erhoben, sie ergeben sich auch logisch aus der Weiterentwicklung der (kommunikativen) Methode. Denn durch den Perspektivenwechsel im Interkulturellen Ansatz kommen nun andere Ziele des Fremdsprachenunterrichts zum Tragen, man sucht die Erklärungen für das Gelingen bzw. Scheitern von Kommunikation verstärkt auch bei anderen Bezugswissenschaften wie der Soziologie, Psychologie, Ethnologie, Kulturwissenschaft oder Kommunikationswissenschaft (vgl. PAULA: 1999, 23-25).

Ein letzter wichtiger Aspekt, der das Deutsche als Fremdsprache in den letzten Jahren verstärkt prägt, ist die zunehmende Bedeutung des Fremd- und Zweitsprachenlernens in Folge der verstärkten (europäischen) Migration, die einerseits durch politische Programme forciert wird, andererseits durch die wirtschaftlich bedingte Zuwanderung aus außereuropäischen Ländern die Staaten verstärkt zwingt, sinnvolle Integrationskonzepte zu entwickeln. Damit sind weitere Referenzwissenschaften wie die Politologie und Migrationsforschung dem bisherigen Spektrum hinzuzufügen. 
Zusammenfassend kann man feststellen, dass für ein modernes Hochschulfach Deutsch als Fremdsprache die Perspektive der Fremdheit die zentrale Frage darstellt, die nach Antworten auch in entsprechenden Bezugswissenschaften sucht.

\section{Entwicklungslinien des Faches Deutsch als Fremdsprache}

Wir wollen die bisherigen Schlussfolgerungen nun noch aus einem historischen Blickwinkel betrachten, denn neue Aspekte, Tendenzen und damit auch Bezugswissenschaften lassen sich für ein Fach an drei Parametern besonders gut ablesen:

1.) an erstmaligen Diskussionen in Fachzeitschriften,

2.) an neuen Konzepten und Arbeitsschwerpunkten internationaler Konferenzen,

3.) am Aufbau von Handbüchern.

\subsection{Fachzeitschriften}

Die wichtigste Diskussion zur Selbstdefinition des Faches Deutsch als Fremdsprache war die so genannte "Strukturdebatte“, die in den neunziger Jahren in der Zeitschrift „Deutsch als Fremdsprache“ geführt wurde. ${ }^{1}$ Man einigte sich bekanntlich auf vier gleichberechtigte Säulen des Faches: auf Linguistik, Literaturwissenschaft, Methodik und Landeskunde, womit eine erste entscheidende Ausweitung der Bezugswissenschaften vorgenommen wurde. Diese vier Säulen wurden in den letzten zwanzig Jahren zumindest um zwei weitere ergänzt, um die Sprachlehr- und Sprachlernforschung und die Sprachenpolitik.

\subsection{Konferenzen}

Die größte und wichtigste internationale Fachveranstaltung für Deutsch als Fremdsprache ist die Internationale Deutschlehrertagung (IDT), die seit 1967 zuerst in einem Zweijahresrhythmus, dann alle drei und heute alle vier Jahre stattfand bzw. stattfindet. Am erstmaligen Auftauchen einzelner Sektionen oder Hauptvorträge kann man die neuen Impulse und Richtungen der Disziplin erkennen (vgl. dazu SORGER: 2010, 404-417):

1971: Österreichisches Deutsch (Plurizentrik)

1974: Lernziel, Individualisierung und Leistungskontrollen, Kontrastivität, Leistungskontrolle

1977: Faktoren der sprachlichen Kommunikation, Lehr- und Lernmittel

1 An der Diskussion beteiligten sich in den Jahren 1992-1999 u. a. Henrici, Koreik, Götze, Suchsland, Königs, Helbig, Neuner, Blei, Hirschfeld, Rösler, Portmann, Altmayer, Glück, Wenzel. 
1980: Motivation und Lernverhalten, Lernstrategien, Lehrerpersönlichkeit 1983: Arbeits- und Sozialformen, Lerneffektivität

1986: Die Erfahrung von Fremdsein, nationale Varianten, Lernerautonomie 1993: Identität und Integration, Kunst, Projektarbeit, Lernpsychologie

1997: Sprachenpolitik/Multimedia

2001: Mehrsprachigkeitsforschung/Portfolio/Fachunterricht/Aktionsforschung 2005: Gehirnforschung, Motivationsforschung, Tertiärsprachenlernen, Qualitätssicherung

2009: CLIL

\subsection{Handbücher}

Neben dem Handbuch Fremdsprachenunterricht (4. Aufl. 2003) gilt wohl unbestritten das Handbuch Deutsch als Fremdsprache 2001 als die beste Orientierung und Grundlage der wissenschaftlichen Disziplin ebenso wie des Praxisfaches DaF. Ende 2010 erscheint eine Neuauflage des Handbuch Deutsch als Fremdsprache und Zweitsprache, in der in 19 Kapiteln die wichtigsten Aspekte des Faches betrachtet werden. Es fällt auf, dass man aufbauend auf den traditionellen Bereichen (linguistische Gegenstände, Rolle der Literatur, Variationen und Sprachkontakt, Fach- und Wissenschaftssprachen, Medien und Lehr-/Lernmaterialien, Leistungsmessung und Leistungskontrolle, Kontrastivität und Sprachvergleich, Landeskunde und kulturwissenschaftliche Aspekte) nun auch verstärkt der Differenzierung in $\mathrm{DaF}$ und DaZ (das Kapitel „Spezifische Bedingungen und Zielsetzungen des DaZ-Unterrichts“), der Fachgeschichte („Entwicklungslinien des Faches“) und der Sprachenpolitik Aufmerksamkeit schenkt. Einen entscheidenden Schwerpunkt legt das Konzept aber auf den Spracherwerb und das Lehren und Lernen in Praxis und Forschung (DaF/DaZ als spezifisches Lehr- und Forschungsgebiet, Spracherwerb und Sprachlernen, Lernen: spezifische Variablen und Faktoren, Lehren - Zielsetzungen und Methoden/Einzelaspekte, Lehrerinnen und Lehrer) wodurch ein entscheidender Perspektivenwechsel vorgenommen wird. Nicht mehr die Sprache ansich steht im Mittelpunkt des fachlichen Interesses, sondern ihre Vermittlung bzw. ihr Erwerb aus der Sicht des Fremdsprachigen. Damit ist die weitere Entwicklungslinie von Deutsch als Fremdsprache vorgezeichnet und die Abgrenzung zur traditionellen (Auslands-) Germanistik klar vollzogen.

\section{Zusammenfassung}

Deutsch als Fremdsprache lässt sich zwar seit dem Mittelalter als Praxisfach nachweisen und wird heute weltweit gelehrt, die wissenschaftliche Disziplin erlebte aber erst etwa in den letzten dreißig Jahren ihre Konsolidierung und schrittweise Abgrenzung zur Germanistik. Politische und gesellschaftliche Veränderungen beeinflussen die 
Motive Deutsch zu lernen nachhaltig und wirken sich dadurch auf das Fach- und Ausbildungsprofil aus. Die neuen Herausforderungen des Faches aber bedingen eine Ausweitung der Bezugswissenschaften, sodass wir Deutsch als Fremdsprache heute als interdisziplinäres Forschungsfach verstehen können. Will man an den Universitäten der Zukunft die Studierendenzahlen halten oder gar ausweiten, so muss sich diese Entwicklung in den nächsten Jahren auch in den universitären Curricula zu Veränderungen und neuen Schwerpunktsetzungen führen.

\section{Literatur:}

AMMON (1989): Ammon, Ulrich. Zur Geschichte der Sprachverbreitungspolitik der Bundesrepublik Deutschland von den Anfängen bis 1985, in: Deutsche Sprache, 1989, 17, 229-263.

AMMON (2001): Ammon, Ulrich. Die Verbreitung des Deutschen in der Welt, in: Helbig, Gerhard/Götze, Lutz/Henrici, Gert/Krumm, Hans-Jürgen (Hg.): Deutsch als Fremdsprache. Ein internationales Handbuch. 2. Halbband. Berlin, New York: Ohne Verlagsangabe, 2001, 1368-1381.

BAUSCH/CHRIST/KRUMM (2003): Bausch, Karl-Richard/Christ, Herbert/Krumm, Hans-Jürgen (Hg.). Handbuch Fremdsprachenunterricht. Tübingen: Francke, 4. Aufl., 2003.

BUNDESREGIERUNG (1985): Bundesregierung. Bericht der Bundesregierung über "Die Stellung der deutschen Sprache in der Welt". [Hrsg: Auswärtiges Amt, Referat für Öffentlichkeitsarbeit], Bonn: Ohne Verlagsangabe, 1985.

EDER (2006): Eder, Ulrike. „Auf die mehrere Ausbreitung der teutschen Sprache soll fúrgedacht werden". Deutsch als Fremd- und Zweitsprache im Unterrichtssystem der Donaumonarchie zur Regierungszeit Maria Theresias und Josephs II. Innsbruck: Studienverlag, 2006.

EUROPÄISCHE KOMMISSION (2006): Europäische Kommission. Die Europäer und ihre Sprachen. Befragung: November - Dezember 2005. Abrufbar unter: http://ec.europa.eu/ public_opinion/archives/ebs/ebs_243_sum_de.pdf (letzte Abfrage am 30.10.2010).

FACHLEXIKON (2010): Barkowski, Hans/Krumm, Hans-Jürgen. Fachlexikon Deutsch als Fremdsprache. Tübingen, Basel: Franke, 2010.

FaDaF: Grundsatzpapier des Fachverbands Deutsch als Fremdsprache zur curricularen Basis der BA/MA-Studiengänge „Deutsch als Fremdsprache“. Abrufbar unter: http://www.fadaf.de/ de/daf_angebote/studieng_nge/grundsatzpapier.pdf (letzte Abfrage am 25.10.2010).

FIEBACH (2010): Fiebach, Constanze. Die Welt lernt Deutsch - aktuelle Erhebung der Lernerzahlen 2010. Abrufbar unter: http://www.goethe.de/ges/spa/dos/daf/ de6139473.htm (letzte Abfrage am 29.10.2010).

GIERSBERG (2009): Dagmar Giersberg. DaF-Studiengänge im Ausland - Berufsbezug statt philologischer Ausrichtung. Abrufbar unter: http://www.goethe.de/ges/spa/dos/daf/ stu/de4611941.htm (letzte Abfrage am 29.10.2010).

GLÜCK (2002): Glück, Helmut. Deutsch als Fremdsprache in Europa vom Mittelalter bis zur Barockzeit. Berlin, New York: de Gruyter, 2002.

GLÜCK (2002a): Glück, Helmut (Hg.). Die Volkssprachen als Lerngegenstand im Mittelalter und in der frühen Neuzeit, Akten des Bamberger Symposions am 18. und 19. Mai 2001. Berlin, New York: de Gruyter, 2002.

HERNIG (2005): Hernig, Marcus. Deutsch als Fremdsprache - eine Einführung. Wiesbaden: Verlag für Sozialwissenschaften, 2005. 
HEXELSCHNEIDER (1980): Hexelschneider, Erhard. Internationale Kulturbeziehungen und Deutsch als Fremdsprache, in: Deutsch als Fremdsprache, 1980, 17, Nr. 3, 133-137.

KRATH (2008): Krath, Stefany. Deutsche Auslandschularbeit: spannende Geschichte mit langer Tradition, in: Bundesverwaltungsamt (Hg.): Zentralstelle für das Auslandsschulwesen. Deutsche Auslandsschularbeit - 40 Jahre ZfA. Köln: Ohne Verlagsangabe, 2008, 20-26.

LEHBERGER (2003): Lehberger, Reiner. Geschichte des Fremdsprachenunterrichts bis 1945, in: Bausch, Karl-Richard/Christ, Herbert/Krumm, Hans-Jürgen (Hg.) (2003): Handbuch Fremdsprachenunterricht. Tübingen: Francke, 4. Aufl., 2003, 609-614.

McCOMB: McComb, Chris. About One in Four Americans Can Hold a Conversation in a Second Language, in: GALLUP NEWS SERVICE. Abrufbar unter: http://www. gallup.com/poll/1825/About-One-Four-Americans-Can-Hold-Conversation-SecondLanguage.aspx (letzte Abfrage am 24.10.2010).

NETZWERK DEUTSCH (2010):Netzwerk Deutsch. Die deutsche Sprache in der Welt. Datenerhebung 2010 Abrufbar unter: http://www.goethe.de/uun/pub/de5759780.htm (letzte Abfrage am 29.10.2010).

PAULA (1999): Sprache und Kultur, in: Buttaroni, Susanna/Paula, Andreas (Hg.): Ehe, Berge und schwarze Katzen. Interkulturelle Komunikation im Fremdsprachenunterricht. Merano/ Meran: Alpha \& Beta Verlag, 1999, 21-62.

ROGGAUSCH (2008): Werner Roggausch. Bologna-Prozess und Germanistik. Abrufbar unter: http://www.daad.ru/wort/wort2008/3_Roggausch_Bologna.pdf (letzte Abfrage am 30.10.2010).

SATORIUS/RUCKTESCHELL (2000): Satorius, Joachim/Ruckteschell, Katharina von. Förderung der deutschen Sprache durch das Goethe-Institut - mit besonderer Berücksichtigung Russlands, in: Ammon, Ulrich (Hg.): Sprachförderung. Schlüssel auswärtiger Kulturpolitik. Frankfurt am Main, Wien [u.a.]: Lang, 2000, 73-82.

SORGER (2010): Der Internationale Deutschlehrerverband im Spannungsfeld von sprachenpolitischen Konzepten und fachlicher Interessensvertretung. Dissertation, Universität Wien, 2010.

StADaF (2006): Ständige Arbeitsgruppe Deutsch als Fremdsprache. Deutsch als Fremdsprache weltweit. Datenerhebung 2005. StaDaF 2005-2006. Abrufbar unter: http://www.goethe. $\mathrm{de} / \mathrm{mmo} / \mathrm{priv} / 1459127-S T A N D A R D$.pdf (letzte Abfrage am 18. 4. 2010). 